PROCE EDINGS OF THE

AMERICAN MATHEMATICAL SOCIETY

Volume 51, Number 1, August 1975

\title{
ZERO DIVISORS IN TENSOR PRODUCTS OF DIVISION ALGEBRAS
}

\author{
LAWRENCE J R RISMAN
}

ABSTRACT. Theorem. If a tensor product of a division algebra $D$ with a quaternion algebra $Q$ is not a division algebra, then either $D$ and $Q$ possess a common quadratic subfield or $D$ contains a splitting field of $Q$ not quadratic over the base field.

The above theorem generalizes a recently published result of Albert's.

Theorem. If the tensor product of two division algebras over a local or a global field $K$ is not a division algebra, then they contain a common extension field of $K$.

Let $K$ be a field. We consider algebras not necessarily finite dimensional over $K$ and their tensor products over $K$. A. A. Albert has proven that two quaternion division algebras have a common quadratic subfield if their tensor product contains zero divisors [1].

We show that a more general result follows by the same arguments.

Theorem 1. Let $D$ be a division algebra over $K$ and $Q$ a quaternion division algebra with center $K$. Suppose $D \otimes Q$ is not a division algebra. Then either $D$ and $Q$ possess a common quadratic extension field of $K$, or $D$ contains a splitting field of $Q$ that is not quadratic over $K$.

Proof. Let $K(u)$ be a subfield of $Q$ which is a separable quadratic extension of $K$. Let $u \rightarrow u^{\prime}$ under the nontrivial automorphism of $K(u)$ over $K$. Let $v \in Q$ with $u v=v u^{\prime}, v^{-1} u v=u^{\prime}$.

Then $u u^{\prime}=a$ and $v^{2}=b$ with $a$ and $b$ nonzero elements of $K$. Let $L=D \otimes K(u)$. If $L$ contains zero divisors, then $D$ contains a subfield isomorphic to $K(u)$, and we are done. Otherwise, $L$ is a division algebra.

By hypothesis $D \otimes Q$ is not a division algebra, and hence contains zero divisors. As a vector space over $L, D \otimes Q=L \oplus L v$. Let $p+q v$ be a zero divisor in $D \otimes Q$ with $p, q \in L$. Clearly $q \neq 0$, since $L$ is a division algebra. After left multiplication by $q^{-1}$, if necessary, we may assume that $q=$ 1. Thus $p+v$ is a zero divisor.

Received by the editors May 20, 1974.

AMS (MOS) subject classifications (1970). Primary 16A40; Secondary 12A65. 
Express $p=c+d u$ with $c, d \in D$. Note that $u$ and $v$ commute with $c$ and $d$. Set $p^{\prime}=v^{-1} p v=c+d u^{\prime}$, so that $p v=v p^{\prime}$. Hence $(p+v)\left(p^{\prime}-v\right)=$ $p p^{\prime}-v^{2}=p p^{\prime}-b$. But $p p^{\prime}-b$ is an element of $L$ and is a zero divisor, hence it is 0 . Hence

$$
\begin{aligned}
b & =p p^{\prime}=(c+d u)\left(c+d u^{\prime}\right)=c^{2}+d^{2} a+c d u^{\prime}+d c u \\
& =c^{2}+d^{2} a+c d\left(u^{\prime}+u\right)+(d c-c d) u .
\end{aligned}
$$

Since $u^{\prime}+u \in K$, the sum of the first three terms is in $D$. Hence $d c-c d=$ 0 , so that $c$ and $d$ commute.

Let $F$ be the subfield of $D$ generated by $c$ and $d$. Then $p+v=c+d u$ $+v$ and $p^{\prime}-v=c+d u^{\prime}-v$ are nonzero elements of $F \otimes Q$. Their product $(p+v)\left(p^{\prime}-v\right)=0$. Hence $F \otimes Q$ contains zero divisors. If $F$ is quadratic over $K$, then $Q$ contains a subfield isomorphic to $F$. In any case, $F$ splits $Q$ and we have the stated results. Q.E.D.

The above theorem has the following alternative statement: If $D \otimes Q$ contains zero divisors, then there is a commutative subfield of one of these algebras whose tensor product with the other algebra contains zero divisors.

It would be interesting to know to what other pairs of algebras either statement of this theorem can be extended. If $K$ is a local or global field, the conclusion of the theorem extends to all pairs of finite dimensional central division algebras over $K$. This is a consequence of class field theory.

Theorem 2. Let $K$ be a local or global field. Let $D_{1}, \ldots, D_{n}$ be division algebras central over $K$ with $d_{i}=$ degree of $D_{i}$. Then $D_{1}, \ldots, D_{n}$ possess a common subfield cyclic over $K$ whose degree is the greatest common divisor $\left(d_{1}, \ldots, d_{n}\right)$. Furthermore $D_{i} \otimes D_{j}$ is a division algebra if and only if $\left(d_{i}, d_{j}\right)=1$. Hence $D_{i} \otimes D_{j}$ is not a division algebra if and only if $D_{i}$ and $D_{j}$ contain a common extension field of $K$.

Proof. This result follows from the structure theorems for the Brauer group of $K$ in [2, Chapter 9]. The case $d_{i}=d_{j}$ is treated in [3, Theorem 5.4], and the same arguments apply here, mutatis mutandum. Q.E.D.

\section{REFERENCES}

1. A. A. Albert, Tensor product of quaternion algebras, Proc. Amer. Math. Soc. 35 (1972), 65-66. MR $45 \# 6855$.

2. - Structure of algebras, rev. printing, Amer. Math. Soc. Colloq. Publ., vol. 24, Amer. Math. Soc., Providence, R. I. , 1961. MR 23 \#A912.

3. Murray Schacher, Subfields of division rings. I, J. Algebra 9 (1968), 451-477. MR 37 \#2809. 